\title{
Design and Implementation of HoWiES for Power Gaining and Power Reduction
}

\author{
Monali V.Bhadane ${ }^{1}$, Anjali M. Patki ${ }^{2}$ \\ ${ }^{1}$ Indira Collage of Engineering, Pune, Maharashtra, India \\ Professor, Indira Collage of Engineering, Pune, Maharashtra, India
}

\begin{abstract}
Continuous active state of WiFi radio to perform real communications is the major reason for entire system power consumption of mobile devices. Many attempts have been made by both industry and academia to integrate ZigBee radios with smartphones for smart home applications and energy savings. A technique is proposed that applies low power ZigBee radio that delegates the operations performed by WiFi. The ZigBee radio is responsible for discovering the presence of WiFi networks and detecting if the AP intends for the device to communicate meanwhile WiFi radio will be turned off when there is no packet to transmit and receive. An effective lightweight encoding and decoding scheme is applied for one way WiFi communication from AP to zigbee. A technique is contributed to reduce the overhead variable message length WiFi-ZigBee packets are used depending on the frequency of type of packets. Through the network simulation in NS2, it is observed that the proposed technique along with enhanced technique reduces the power consumption and overhead in mobile devices with increase energy gain.
\end{abstract}

Keywords: WiFi, Message delivery Scheme, NS2, zigbee, HoWiEs

\section{Introduction}

WiFi radio is one of the mostly used wireless communication interfaces in mobile devices, and is a major source of energy consumption affecting user experience. ZigBee radio is designed for low power communication working on $2.4 \mathrm{GHz}$ ISM band, which coincides with most of theWiFi standards. Nowadays, increasingly attempts have been made by both industry and academic institutes to integrate ZigBee radios with smart phones for smart home applications and energy savings. Indeed, in system implementation, a ZigBee radio can be directly connected to a mobile device via USB interface. With the unveiling of the first Android phone with ZigBee capability, and due to its usefulness in many areas, it is believed that ZigBee will become a standard interface in mobile devices in the near future. Three significant energy saving opportunities for $\mathrm{WiFi}$ stations.

Opportunity 1 - scanning state: WiFi stations in scanning state constantly iterate through all the channels to search for available WiFi networks.

Opportunity 2 - standby state: As introduced in $\mathrm{x} 2.1$, in standby state, a CAM (Constantly Awake Mode) station keeps its WiFi radio on all the time, and a PSM (Power Save Mode) station puts its WiFi radio in a low-power state most of the time when there is no traffic, and periodically wakes up the radio to receive and check AP beacons for buffered packets.

Opportunity 3 - energy waste due to wakeup contention: When multiple PSM stations working at the same channel and associated either with the same AP or with multiple colocated APs, are waken up to receive buffered packets at the same time, the contention between the stations will force them to stay active with out performing any real communications. This idle waiting could further causes about up to 4 times more energy consumption. Motivated by these research results, proposed approach applies the scheme to wake up standby $\mathrm{WiFi}$ radios to avoid these energyexpensive wakeup contentions.

It is a challenging to design a message encoding/decoding scheme that forms/detects messages by using/interpreting the combinations of alphabet characters, since the existence of normal WiFi packets will make the scheme suffer from detection errors. To address this challenge, it design a selfcorrecting message encoding/decoding scheme that can effectively reduce skewed messages due to the interference from normal WiFi packets. Second, instead of focusing on studying the feasibility to enable $\mathrm{WiFi}$ radios to communicate with Zig-Bee radios, it is targeted at designing and implementing a practical system that saves WiFi energy for mobile devices in different aspects by using proposed WiFi-ZigBee message delivery scheme. Although ZigBee radios are more power efficient than $\mathrm{WiFi}$ radios, it is not trivial to design and implement a system that saves $\mathrm{WiFi}$ energy in several aspects with the assistance of ZigBee radios. For example, an active ZigBee radio consumes comparable amount of energy to a WiFi radio that is in PSM standby. To save the energy a $\mathrm{WiFi}$ radio spends during standby, we have managed to get the ZigBee interface to synchronize with the wireless AP, and to duty-cycle the ZigBee the interface to reduce its power Consumption.

To reduce WiFi power consumption a technique is proposed to delegate those $\mathrm{WiFi}$ operations to a low power ZigBee radio. In this case, $\mathrm{WiFi}$ radio will be turned off when there is no packet to transmit and receive, and the ZigBee radio is responsible for discovering the presence of WiFi networks and detecting if the AP intends for the device to communicate. This way, the significant power consumptions on $\mathrm{WiFi}$ radio in those scenarios can be reduced to reasonably low power consumptions of ZigBee radio.

Using zigbee proposed work design and implement HoWiES, a system that utilizes ZigBee radio to perform WiFi scanning and periodic AP beacon checking for the WiFi interface in the same device. The scenario is that a mobile device keeps 


\section{International Journal of Science and Research (IJSR) \\ ISSN (Online): 2319-7064}

Index Copernicus Value (2013): 6.14 | Impact Factor (2015): 6.39

its WiFi interface offline, while using its ZigBee radio to discover WiFi networks (during WiFi scanning) and to check if the associating AP has buffered the device's packets (during WiFi PSM standby). Encoding/decoding scheme for WiFi - Zigbee communication.

Given that it is not possible for ZigBee to decode WiFi packets directly, it is necessary to build a channel, through which a WiFi AP can convey information to ZigBee radio in mobile devices. Since the AP does not need feedbacks from the mobile device in the intended scenarios, a unidirectional channel from WiFi AP to ZigBee radio is sufficient. Fortunately, the same frequency band occupied by both $\mathrm{WiFi}$ and ZigBee allows ZigBee radio to sample background energy of WiFi transmission, which enables WiFi AP to convey information to ZigBee radio by using different $\mathrm{WiFi}$ transmission patterns.

Using a simple coding scheme, proposed system can create a unidirectional communication channel in which an AP can deliver thousands of messages to ZigBee radios. When ZigBee decodes the information transmitted through the side channel, it either ignores the message if it is not for the device, or wakes up the $\mathrm{WiFi}$ radio for Communication. Proposed power saving Protocols specifically target the three significant $\mathrm{WiFi}$ energy saving opportunities in $\mathrm{WiFi}$ scanning, standby and wakeup respectively.

In this work, we design and implement HoWiES, a system that utilizes ZigBee radio to perform $\mathrm{WiFi}$ scanning and periodic AP beacon checking for the $\mathrm{WiFi}$ interface in the same device. The scenario is that a mobile device keeps its WiFi interface offline, while using its ZigBee radio to discover WiFi networks (during WiFi scanning) and to check if the associating AP has buffered the device's packets (during WiFi PSM standby). Given that it is not possible for ZigBee to decode $\mathrm{WiFi}$ packets directly, it is necessary to build a channel, through which a WiFi AP can convey information to ZigBee radio in mobile devices. Since the AP does not need feedbacks from the mobile device in the intended scenarios, a unidirectional channel from WiFi AP to $\mathrm{ZigBee}$ radio is sufficient.Fortunately, the same frequency band occupied by both WiFi and ZigBee allows ZigBee radio to sample background energy of WiFi transmission, which enables WiFi AP to convey information to ZigBee radio by using different $\mathrm{WiFi}$ transmission patterns. We demonstrate that, by using a simple coding scheme, our system can create a unidirectional communication channel in which an AP can deliver thousands of messages to ZigBee radios. When ZigBee decodes the information transmitted through the side channel, it either ignores the message if it is not for the device, or wakes up the WiFi radio for communication.

\section{Overhead Reduction through Variable Message Length}

All WiFi-ZigBee messages have the same length (i.e., use the same number of packets to encode different messages). A promising way to increase the efficiency of the message delivery scheme and to reduce message delivery overheads variable message length is used. Hence a technique is contributed that enhances the proposed protocol by applying less packets to encode those frequently used messages and more packets to encode those rarely used messages

\section{Architecture}

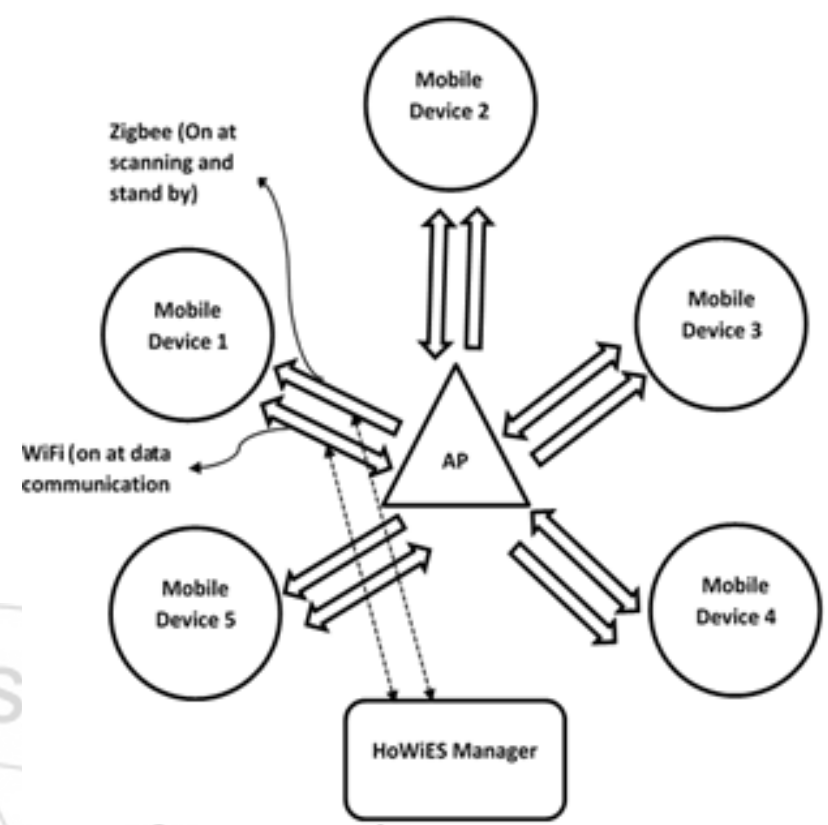

Figure 1: Architecture of System

\section{Block Diagram}

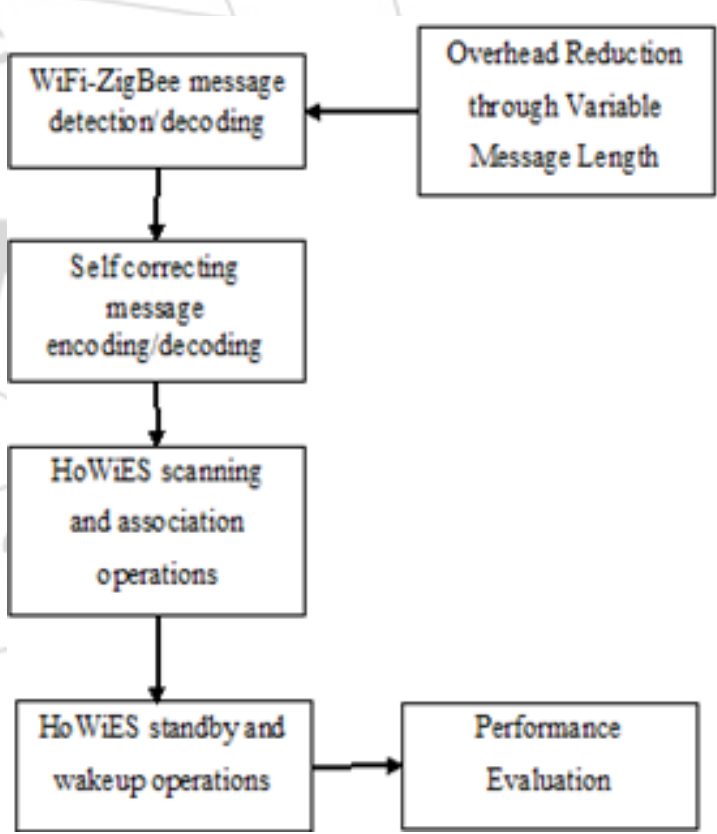

Figure 2: Block diagram of System

\section{Software Requirements}

Simulator :NS version 2.35

Language : TCL and AWK script

Operating System :Windows7(Cygwin)/ Ubuntu 12.04

\section{Simulation}


International Journal of Science and Research (IJSR)

ISSN (Online): 2319-7064

Index Copernicus Value (2013): 6.14 | Impact Factor (2015): 6.39

\begin{tabular}{|l|l|}
\hline SIMULATOR & Network Simulator 2 \\
\hline NUMBER OF NODES & Random \\
\hline AREA & $500 \mathrm{~m} \times 500 \mathrm{~m}$ \\
\hline COMMUNCATION RANGE & $250 \mathrm{~m}$ \\
\hline INTERFACE TYPE & PhyWirelessPhy \\
\hline MAC TYPE & 802.11 \\
\hline QUEUE TYPE & DroptailPriorityQueve \\
\hline QUEUE LENGTH & 200 Packets \\
\hline ANIENNA TYPE & Omin Antenna \\
\hline PROPAGATION TYPE & TwoRayGround \\
\hline ROUTING AGENT & ADDV \\
\hline TRANSPORT AGENT & UDP \\
\hline APPLICATION AGENT & CBR \\
\hline SIMULATION TIME & 50 seconds \\
\hline
\end{tabular}

The capacity of a message delivery scheme, which is the total amount of numbers that can be encoded, is bl. Here R, 1 , $\mathrm{b}$ and $\mathrm{A}$ are fixed and shared between WiFi and ZigBee radios.

ZigBee radios use to detect and decode WiFi-ZigBee messages. ZigBee radios detect WiFi-ZigBee messages by continuously sampling background energy with a frequency $\mathrm{H}$. If a sample' s energy reading is greater than a threshold $\mathrm{E}$, the sample is a "positive" sample, otherwise it is a "negative" sample.

\subsection{WiFi-ZigBee message delivery scheme: Self} correcting message encoding/decoding

Input: Background (packet) interference

Output: Correct value of a message

The alphabet $\mathrm{A}=\{\mathrm{S} 1, \mathrm{Sb}\}$ is divided into $\mathrm{p}$ sub-alphabets as $\mathrm{A} 1=\quad\{\mathrm{S} 1, \mathrm{Sp}+1, \mathrm{~S} 2 \mathrm{p}+1, .\},. \quad \mathrm{A} 2=\{\mathrm{S} 2, \quad \mathrm{Sp}+2, \quad \mathrm{~S} 2 \mathrm{p}+2, .\}$, $A p=\{S p, S 2 p, S b g\}$ To encode a message, a WiFi radio uses packet sizes in one randomly chosen sub-alphabet. To decode a message, a ZigBee radio gets the sizes of all the message packets. If all the sizes are from the same subalphabet, the ZigBee radio can calculate the message value directly. Otherwise, it indicates that there were background interferences happened to the message packets. In this case, the ZigBee radio first identify the correct sub-alphabet (notated as Ac) as the sub-alphabet to which the majority packet sizes belong. Then it converts each of those packet sizes that are not in Ac to the value in Ac that is immediately smaller than the current wrong size. With the self correcting scheme, ZigBee radios can still extract the correct value of a message with high possibility even if background interferences exist.

\subsection{HoWiES scanning and association operations}

Input: WiFi turnoff, ZigBee turn on

Output: Notification from Zigbee about presence, Wifi on and association to $\mathrm{AP}$

During the scanning process, a HoWiES client determines whether to turn on its $\mathrm{WiFi}$ radio and associate to an $\mathrm{AP}$ based on the numbers encoded in theWiFi-ZigBee messages received. For example, to allow mobile devices to be able to determine whether to turn on WiFi radios and associate to APs based on whether the WiFi network is encrypted, the system operator can let open APs encode number 1 in their network advertisement WiFi-ZigBee messages while encrypted APs encode number 2 in the messages. In this case, mobile devices can selectively turn on their WiFi radios based on whether the encountered networks is encrypted.

Upon detecting an advertisement message (Op.2), the ZigBee component notifies the HoWiES manager about the presence of a WiFi network and the scale of the WiFi signal strength calculated based on the energy samples of the message (Op.3). The HoWiES manager turns on the WiFi radio if the WiFi network meets the device' s needs (Op.4). The WiFi radio sends an association request, indicating that the request issuer is HoWiES-capable, to the AP based on the information in the WiFi beacons (Op.5 and 6). If the association succeeds, the AP chooses a number from its 


\section{International Journal of Science and Research (IJSR) \\ ISSN (Online): 2319-7064}

Index Copernicus Value (2013): 6.14 | Impact Factor (2015): 6.39

message number pool to assign to the newly associated client as its HoWiES ID, and puts this ID in the association response (Op.7). Finally, the WiFi component extracts the ID from the association response and send it to the $\mathrm{ZigBee}$ radio via the HoWiES manager (Op.8-9).

\subsection{HoWiES standby and wakeup operations}

Input: WiFi turnoff, ZigBee turn on Output: Message detection by Zigbee, Wifi turn on

The HoWiES manager keeps monitoring the WiFi traffic on the mobile device (Op.1). On detecting that theWiFi radio has been idle for a predefined amount of time, the HoWiES manager notifies the WiFi radio to go into HoWiES standby state (Op.2). Then the WiFi radio informs the AP that it will switch to the HoWiES standby state and then turns itself off for energy savings (Op.3). Right after notifying the WiFi component to switch to HoWiES standby, the HoWiES manager turns on the ZigBee radio for WiFi-ZigBee message listening during standby (Op.20). With this protocol, WiFi radios in HoWiES standby devices do not need to switch to active periodically to check beacons for buffered packets. Instead, they can just sleep all the time till the ZigBee radio detects wakeup messages sent from the AP.

Once the AP has buffered incoming packets for a HoWiES standby client, it wakes up the client by sending out a WiFiZigBee message that encodes the HoWiES ID assigned to the client in the association process (Op.5). If the buffered packets are broadcast/multicast packets, a common number, instead of the HoWiES ID, is encoded in the message. If there are multiple clients that have buffered packets, the AP wakes them up one by one in a FIFO manner. The ZigBee component informs the HoWiES manager about the buffered packets if it detects the number encoded by a WiFi-ZigBee message matches the device' s HoWiES ID (Op.6). Then the HoWiES manager turns on the WiFi radio (Op.7), which in turn gets the buffered packets from the AP (Op. 8-9).

\subsection{Overhead Reduction through Variable Message Length}

Input: WiFi-ZigBee messages

Output: variable message length for WiFi-ZigBee messages

All WiFi-ZigBee messages have the same length (i.e., use the same number of packets to encode different messages). A promising way to increase the efficiency of the message delivery scheme and to reduce message delivery overheads variable message length is used. Hence a technique is contributed that enhances the proposed protocol by applying less packets to encode those frequently used messages and more packets to encode those rarely used messages.

\section{Performance Evaluation}

Throughput:

The throughput is the number of bits successfully transmitted per second.

1) Overhead on network throughput

This metric represents the amount of control packet transmissions that affects the network throughput.

2) Overhead on AP performance
This metric represents the amount of control packet transmissions that affects the AP delivery performance.

3) Energy gain

It represents the amount of energy saved due to the use of power saving protocol

\section{Result (Simulation Screenshots)}

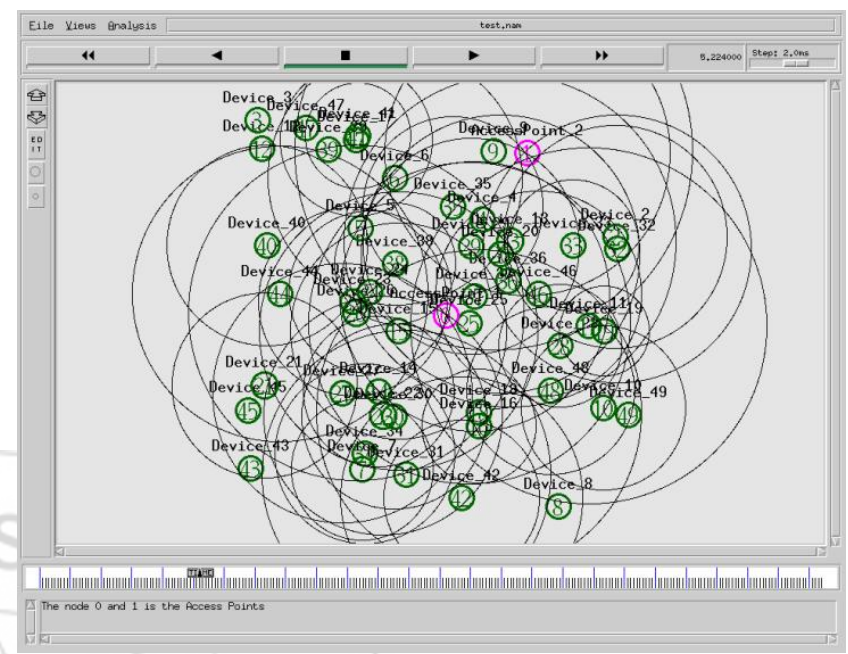

Figure 3: WiFi-ZigBee message delivery scheme: WiFiZigBee message decoding

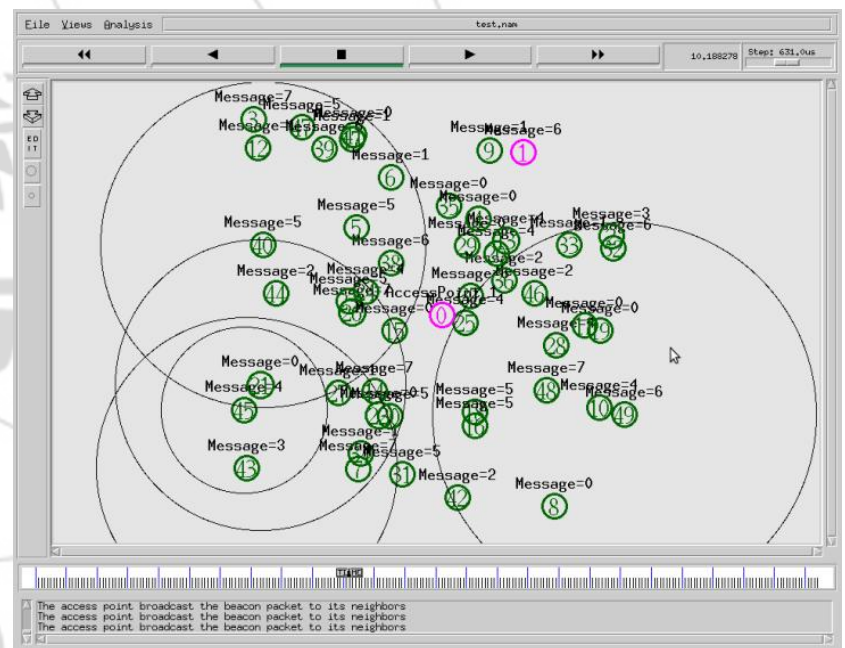

Figure 4: HoWiES Scanning and Association Operations

\section{Result (Comparative Graph)}


International Journal of Science and Research (IJSR)

ISSN (Online): 2319-7064

Index Copernicus Value (2013): 6.14 | Impact Factor (2015): 6.39

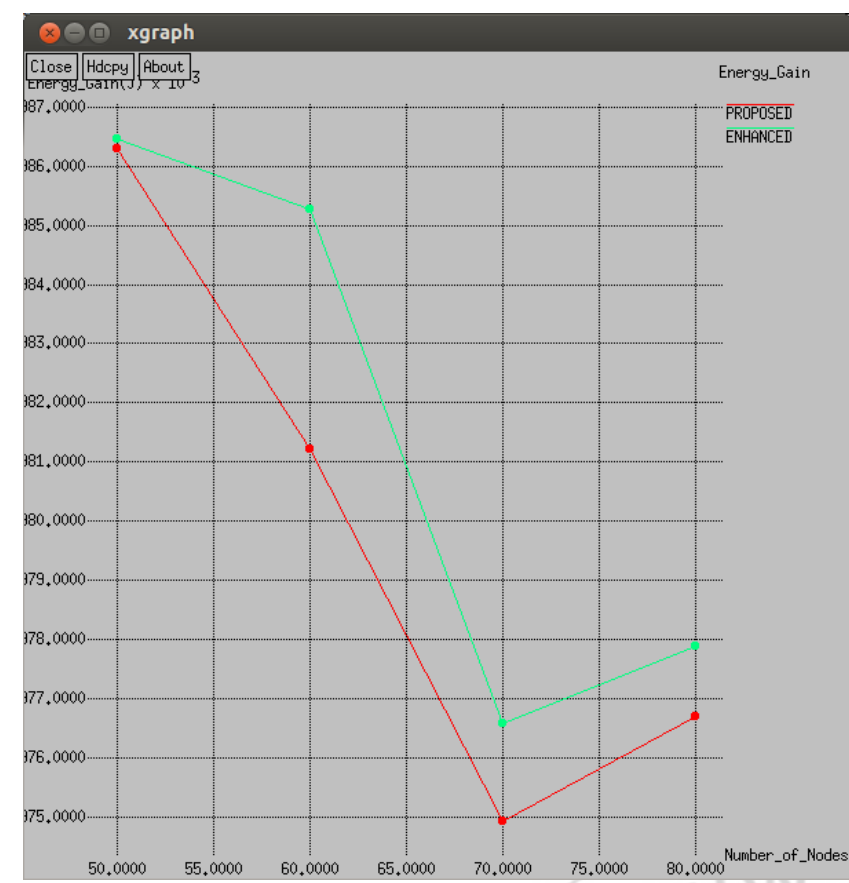

Figure 5: Graph of Energy Gain comparision proposed and Enhanced System

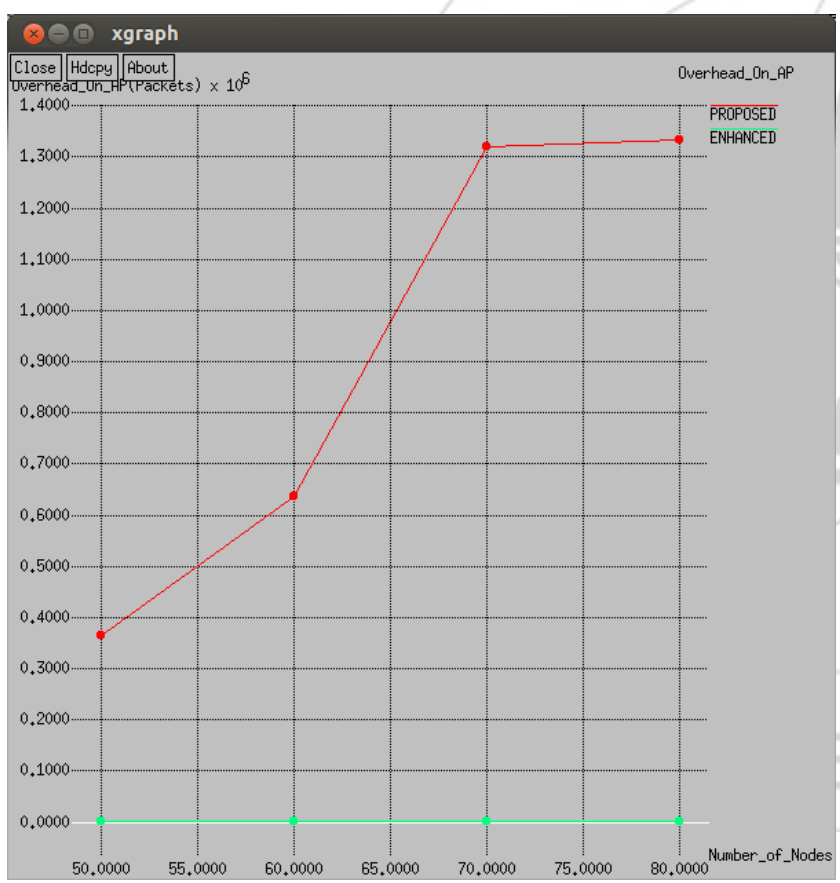

Figure 6: Graph of Overhead on Access Point: Comparision proposed and Enhanced System

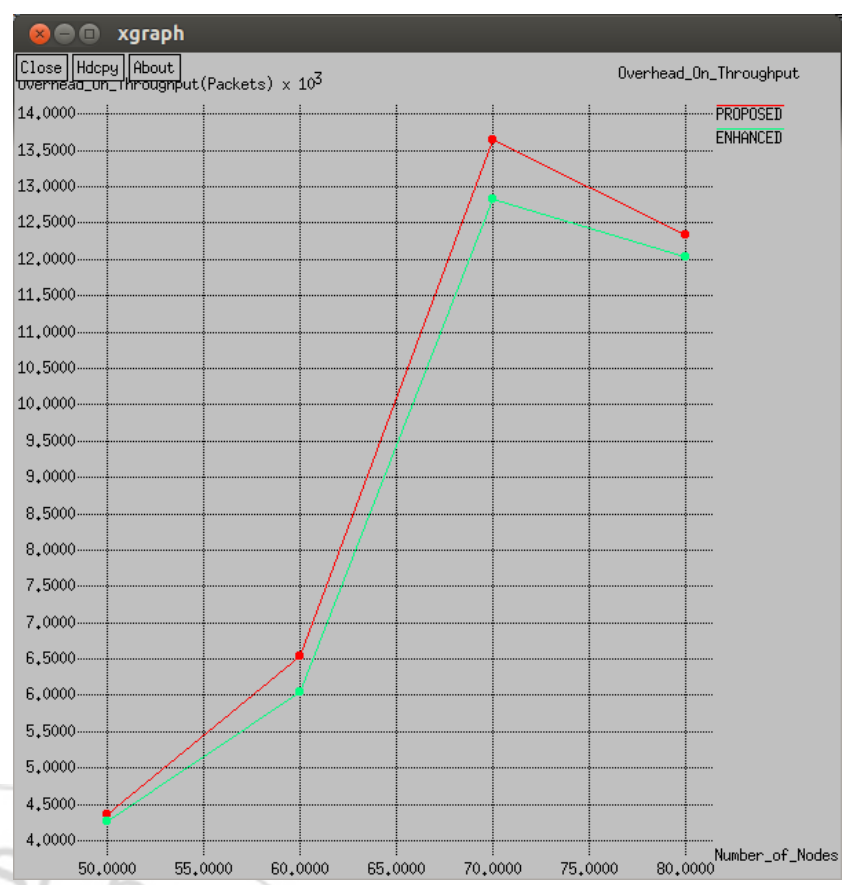

Figure 7: Graph of Overhead on Throughput comparision proposed and Enhanced System

\section{Conclusion}

Proposed work has been simulated successfully by applying low power ZigBee radio that delegates the operations performed by WiFi. The ZigBee radio discovers the presence of WiFi networks and detects the AP intends for the device to communicate meanwhile $\mathrm{WiFi}$ radio turned off when there is no packet to transmit and receive. Encoding and decoding scheme has been lightweight one way WiFi communication from AP to zigbee. Contributed technique reduces the overhead with variable message length WiFi-ZigBee packets. Proposed along with enhanced technique reduces the power consumption and overhead in mobile devices with increase energy gain.

\section{References}

[1] H. Han, Y. Liu, G. Shen, Y. Zhang, and Q. Li, "DozyAP: powerefficient Wi-Fi tethering," in MobiSys, 2012.

[2] Y. Zhang and Q. Li, "HoWiES: A holistic approach to ZigBee assisted WiFi energy savings in mobile devices," in INFOCOM, 2013.

[3] F. Xu, Y. Liu, Q. Li, and Y. Zhang, "V-edge: Fast Selfconstructive Power Modeling of Smartphones Based on Battery Voltage Dynamics,"in USENIX NSDI, 2013.

[4] Y. Zhang, X. Wang, X. Liu, Y. Liu, L. Zhuang, and F. Zhao,"Towards Better CPU Power Management on Multicore Smartphones,"in Hotpower, 2013.

[5] Y. Zhang, C. C. Tan, and Q. Li, "CacheKeeper: A System-wide Web Caching Service for Smartphones," in Ubicomp, 2013.

[6] W. Li, Y. Cui, X. Cheng, M. Al-Rodhaan, and A. AlDhelaan, "Achieving Proportional Fairness via AP Power Control in Multi-Rate WLANs." IEEE Transactions on Wireless Communications, 2011.

[7] E. Rozner, V. Navda, R. Ramjee, and S. K. Rayanchu, "NAPman: network-assisted power management for wifi devices," in MobiSys, 2010. 


\section{International Journal of Science and Research (IJSR) \\ ISSN (Online): 2319-7064}

Index Copernicus Value (2013): 6.14 | Impact Factor (2015): 6.39

[8] J. Manweiler and R. R. Choudhury, "Avoiding the rush hours: WiFi energy management via traffic isolation," in MobiSys, 2011.

[9] IEEE-SA, IEEE Std 802.15.4-2006.

[10] Texas Instruments News Center, TI demonstrates world's first Android development platform bringing ZigBee and ZigBee RF4CE to Smartphones and Tablets, 2011.

[11]R. Zhou, Y. Xiong, G. Xing, L. Sun, and J. Ma, "ZiFi: wireless LAN discovery via ZigBee interference signatures," in MobiCom, 2010.

[12]T. Jin, G. Noubir, and B. Sheng, "WiZi-Cloud: Applicationtransparent dual ZigBee-WiFi radios for low power Internet access," in Infocom, 2011.

[13] TazTag, TPH-ONE, 2012, http://www.taztag.com/PR TPH-ONE revMD V4.pdf.

[14] K. Chebrolu and A. Dhekne, "Esense: communication through energy sensing," in MOBICOM, 2009.

[15] IEEE-SA, IEEE Std 802.11-2007.

[16] A. Rahmati and L. Zhong, "Context-for-wireless: contextsensitive energy-efficient wireless data transfer," in MobiSys, 2007.

[17]A. J. Nicholson and B. D. Noble, "BreadCrumbs: forecasting mobile connectivity," in MobiCom, 2008.

[18] G. Ananthanarayanan and I. Stoica, "Blue-Fi: enhancing Wi-Fi performance using bluetooth signals," in MobiSys, 2009.

[19]E. Shih, P. Bahl, and M. J. Sinclair, "Wake on wireless: : an event driven energy saving strategy for battery operated devices," in MobiCom, 2002.

[20] N. Mishra, K. Chebrolu, B. Raman, and A. Pathak, "Wake-on- WLAN," in WWW, 2006.

[21] Y. Agarwal, R. Chandra, A. Wolman, P. Bahl, K. Chin, and R. K. Gupta, "Wireless wakeups revisited: energy management for VOIP over WiFi smartphones," in MobiSys, 2007.

[22]X. Zhang and K. G. Shin, "E-MiLi: energy-minimizing idle listening in wireless networks," in MobiCom, 2011.

[23]H. Javaheri and G. Noubir, "iPoint: A PlatformIndependent Passive Information Kiosk for Cell Phones," in SECON, 2010.

[24] H. Falaki, R. Mahajan, S. Kandula, D. Lymberopoulos, R. Govindan, and D. Estrin, "Diversity in smartphone usage," in MobiSys, 2010.

[25] A. Shye, B. Scholbrock, G. Memik, and P. A. Dinda, "Characterizing and modeling user activity on smartphones: summary," in SIGMETRICS, 2010.

[26] Bluetooth SIG, BLUETOOTH SPECIFICATION Version 4.0, 2010.

[27] IETF Network Working Group, RFC-1191.

[28] J. Liu and L. Zhong, "Micro power management of active 802.11 interfaces," in MobiSys, 2008.

[29] libpcap library, http://www.tcpdump.org/.

[30]M. Dong and L. Zhong, "Self-constructive high-rate system energy modeling for battery-powered mobile systems," in MobiSys, 2011.

[31] MonsoonSolutions, www.msoon.com/LabEquipment/Pow erMonitor 